\title{
Graft Status
}

National Cancer Institute

\section{Source}

National Cancer Institute. Graft Status. NCI Thesaurus. Code C135522.

The condition of the donated organ or tissue. 